\title{
Syntax and the Chomskyan Revolution
}

\author{
Sudeep SD \\ Assistant Professor of English \\ Government Arts and Science College, Nadapuram \\ Calicut, Kerala, India \\ sudeepdamodar@gmail.com
}

Sounds make up morphemes, morphemes make up words, and words come together in sentences; in linguistics, the study of how words come together in sentences is called syntax.

The crucial insight is that forming sentences is not a mere matter of placing words in order one at a time. Rather, just as the phonemic/phonetic distinction underlies the mere assemblage of sounds, and just as conglomerations of morphemes underlie the surface rendition of words, in syntax there is what lies beneath and what is on the surface.

The history of studying syntax is relatively new. The study of phonology and morphology was well established by the 1920s. However, the leading scholars assumed that each language was an independent growth, rooted partly in its speakers' culture and partly in chance.

This was in part because anthropologists had played a large role in setting the terms of the new discipline. Edward Sapir (1884-1939) and Franz Boas (1858-1942), for example, were ardent proponents of the validity of all human cultures, rejecting earlier conceptions of the world's peoples as rankable on a scale of "development." 
Because language is related to culture, this conditioned an approach to languages as cultural features, learned via the same cognitive processes as all other human activities.

This was also in part because psychology at the time was dominated by behaviorism, which stipulated that human behavior is all learned. Language was thought to be just a matter of an arbitrary, chance conglomeration that one picked up, not a matter of any kind of underlying system. Therefore, linguistic analysis focused on describing the phonemes and morphemes of a language and described how they were placed in order.

However, starting with his work as an undergraduate at the University of Pennsylvania in the 1940s, Noam Chomsky (1928-) began to develop a theory under which all languages have a basic syntactic configuration that we are mentally hardwired to learn and use.

To support this theory, the task of the linguist is to explain how languages that seem so different can be products of this species-wide mental configuration. Chomsky first inaugurated this idea in what was published in 1957 as Syntactic Structures. Within 10 years, this approach to syntax became the dominant one in linguistics, and it is taught universally today.

One of Chomsky's foundational concepts was that syntax is a module of the generation of a sentence that is distinct from its meaning (i.e., its semantics). Earlier, it was generally thought that expressing meaning and syntax were more or less the same process.

Chomsky called attention to facts such as that The dog bit the man and The man was bitten by the dog have the same meaning but different syntax.

Also, a sentence that is syntactically well-formed can be semantically meaningless, such as Colorless green ideas sleep furiously. This sentence is termed grammatical albeit nonsensical. 
Chomsky proposed therefore that there is a mental level that generates grammatical sentences, with their meaning being a separate generative process.

Chomsky's basic idea was that this process is innate, and he called it universal grammar. One of Chomsky's crucial insights was that children do not make all the mistakes that we would expect them to.

For example, to make a sentence into a question, in English we change the order of the subject and the auxiliary, such as:

The man is tall. Is the man tall?

Thus we might think that the rule English speakers follow is simply "switch the order of the subject and the auxiliary."

But to make the sentence The man who is tall is sad into a question, we say Is the man who is tall sad?

Is appears twice in the sentence, but we do not put the first is up front and say Is the man who tall is sad?

This is because who is tall is nested within the sentence as a kind of subsentence. We are questioning the sentence, not the subsentence, and thus we extract the second is.

No one teaches children such a thing — and yet children do not start out trying sentences like Is the man who tall is sad? Chomsky argues that this is because we are born with an innate mental configuration to learn and produce language with this kind of nested structure.

That is, syntax is not a mere matter of learning the order of elements.

Syntax is a matter first of constituency and second of a nested, or hierarchical, structure that the elements are slotted into. 
Chomskyan syntax has worked out a formal representation of how sentences are represented in the brain in this structure-dependent fashion. The representation is in the form of "trees," in which words occur at the ends of the "branches."

The tree begins with a basic distinction between an entity and a predication, traditionally called subject and predicate: Bill (subject) walked away (predicate). The top node of the tree is rendered as $\mathrm{S}$, for sentence. The subject is rendered as $\mathrm{N}$ for noun and the predicate as $\mathrm{V}$ for verb.

However, a subject can consist of more than just a noun. The noun can be modified by an adjective: Black cats. The noun can have an article: The black cat. For this reason, the subject node in our tree needs room for more than just a noun alone. The node is, instead, specified as for a noun phrase, abbreviated on the tree as NP. It has a hierarchical structure.

In the same way, a verb as often as not is followed by an object (Bill saw Jim) or modified by an adverb (Bill walked slowly).

The tree has a verb phrase node (abbreviated as VP) next to the noun phrase one as branches of the S node, and then the verb itself and the object noun are branches of the VP node.

NP structure can also occur under the VP node: Bill saw the little man would "tree" with the VP having two branches: one the verb saw and the other an NP, which would contain the little man.

Thus all nouns in a sentence, subject or object, are potentially NPs, with the same possible branching into definite article, adjective, and so on, in a hierarchical fashion.

The idea is that this is not simply a way to diagram a sentence but that we are born with an innate propensity to process and produce language as driven by this particular tree structure. 
Motivation for the idea that we are born with a sensitivity to this kind of structure becomes clearer when we look further at how trees work.

A verb can also take a complement that is, in effect, a separate sentence: In Sue said that Bill lied, the phrase that Bill lied is a sentence nested within the main one.

1. On our tree, we indicate this with a node called the complement phrase, abbreviated $\mathrm{CP}$, which comes under the VP.

2. The $\mathrm{CP}$ branches into a complement node known as a complementizer, abbreviated as $\mathrm{C}$, which takes the that, and then an S node for a whole new sentence: Bill lied.

This brings us back to The man who is tall is sad. Here, who is tall is a sentence of its own, termed a relative clause.

It fits into the tree as a CP as well, but who is the subject of this "sentence," and so the complement node itself hangs empty.

That empty node is a hint that this is not just the parsing or diagramming that you may have learned in school. This is a different kind of formalism.

Under the Chomskyan conception, a child would first hear a sentence and know, on a certain level-literally be genetically programmed to readily understand, although not able to put it in so many words - that there are possible sentences within sentences.

This happens even if the sentence heard is full of empty nodes, like that hanging complementizer in the last example. The child will hear the second auxiliary in The man who is tall is sad as higher, although the child has not been told this. 
The Chomskyan revolution was to study syntax as something autonomous from meaning and semantics and to propose that there is an innate capacity for learning, processing, and producing syntax of a particular kind that is universal to our species. 


\section{Reference}

Akmajian et al. (2010); Aronoff and Rees-Miller (2001); Auroux et al. (2000-2006); Brown (2006); Everett (2012); Koerner and Asher (1995); O’Grady et al. (2011); Pinker (1994).

Akmajian, A., R.A. Demers, A.K. Farmer and R.M. Harnish (2010) Linguistics: An Introduction to Language and Communication, 6th edition. Cambridge, MA: MIT Press. Allan, K. (2010 [2007]) The Western Classical Tradition in Linguistics, 2nd expanded edition. London: Equinox. Apollonius Dyscolus (1981) The Syntax of Apollonius Dyscolus. Transl. Fred W. Householder. Amsterdam: John Benjamins.

Aronoff, M. and J. Rees-Miller (eds) (2001) The Handbook of Linguistics. Oxford and Malden, MA: Blackwell.

Auroux, S., E.F.K. Koerner, H.-J. Niederehe and K. Versteegh (eds) (2000-2006) History of the Language Sciences. 3 vols. Berlin: Walter de Gruyter.

Bloomfi eld, L. (1933) Language. New York: Holt, Rinehart and Winston. Brown, E.K. (General editor) (2006) Encyclopedia of Languages and Linguistics, 2nd edition. 14 vols. Oxford: Elsevier.

Chomsky, N. (1957) Syntactic Structures. The Hague: Mouton. 